\title{
Multiple Moles Mimicking Malignancy: A Rare Case of Serous Cystadenofibroma
}

\author{
${ }^{1}$ Deepti Sandeep Shrivastava, ${ }^{2}$ Kalyani S Mahajan, ${ }^{3} \mathrm{C}$ Hariharan, ${ }^{4}$ Priyakshi Chaudhary
}

\begin{abstract}
Ovarian cystadenofibroma is a rare benign ovarian tumor that contains both epithelial and fibrous stromal components. The external appearance of cystadenofibroma is complex; cystic-tosolid-appearing masses malignant and aggresive looking, put the gynecologist in dilemma for radical surgical management. No clearcut guidelines available in literature for its management in an infertile woman.
\end{abstract}

Keywords: Multiple moles, Mimicking malignancy, Serous cystadenofibroma.

How to cite this article: Shrivastava DS, Mahajan KS, Hariharan C,Chaudhary P. Multiple Moles Mimicking Malignancy: A Rare Case of Serous Cystadenofibroma. J South Asian Feder Obst Gynae 2016;8(1):71-73.

Source of support: Nil

Conflict of interest: None

Date of received: 20 November 2015

Date of acceptance: 14 February 2016

Date of publication: March 2016

\section{INTRODUCTION}

Primary infertility may have varied etiology. Ovarian cystadenofibroma is very rarely found to be associated with it. They are uncommon to the extent of $\sim 1.7 \%$ of all benign ovarian tumors. ${ }^{1}$ It is an uncommon epithelial tumor where fibrous stroma is a dominant component of the neoplasm.

Hereby, we are reporting a case where we did the routine diagnostic hysterolaparoscopy for primary infertility with polycystic ovarian disease but, to our surprise, there were multiple mole-like cysts, mimicking malignancy and, later on histopathology, were diagnosed as cystadenofibroma which has got rare malignant potential. It is well-advised to be aware of the possibility of a cystadenofibroma before selecting an aggressive surgical approach in young patients.

\footnotetext{
${ }^{1}$ Professor and Head, ${ }^{2,4}$ Resident, ${ }^{3}$ Professor

${ }^{1-3}$ Department of Obstetrics and Gynecology, Jawaharlal Nehru Medical College, Wardha, Maharashtra, India

${ }^{4}$ Department of Obstetrics and Gynecology, Acharya Vinoba Bhave Rural Hospital, Wardha, Maharashtra, India
}

Corresponding Author: Deepti Sandeep Shrivastava, Professor and Head, Department of Obstetrics and Gynecology, Jawaharlal Nehru Medical College, Sawangi, Wardha, Maharashtra, India Phone: 09860817801, e-mail:deepti_shrivastava69@yahoo.com

\section{CASE REPORT}

A 25 years old nulliparous woman presented with irregular menses and inability to conceive since 4 years. Pelvic examination was normal. Husband's basic investigations for infertility were done which were not significant. Her routine blood investigations were done which were within normal limits.

Ultrasound revealed bilateral polycystic, congested and bulky ovaries.

Diagnostic hysterolaparoscopy was performed and multiple white and brown vesicular structures were detected in peritoneal cavity. So also multiple vesicular structures resembling bunches of grapes of varied colors like hemorrhagic, brownish, black and transparent along with multiple free floating transparent cysts were detected in pelvic cavity. Some vesicles were studded into the omentum and other peritoneal surfaces which could detach easily on touch by grasper. The picture gave a look of aggressive malignancy.

Differential diagnosis of hydatid cyst, endometriotic cyst, benign ovarian cysts and malignant ovarian tumors was kept in mind (Figs 1A and B).

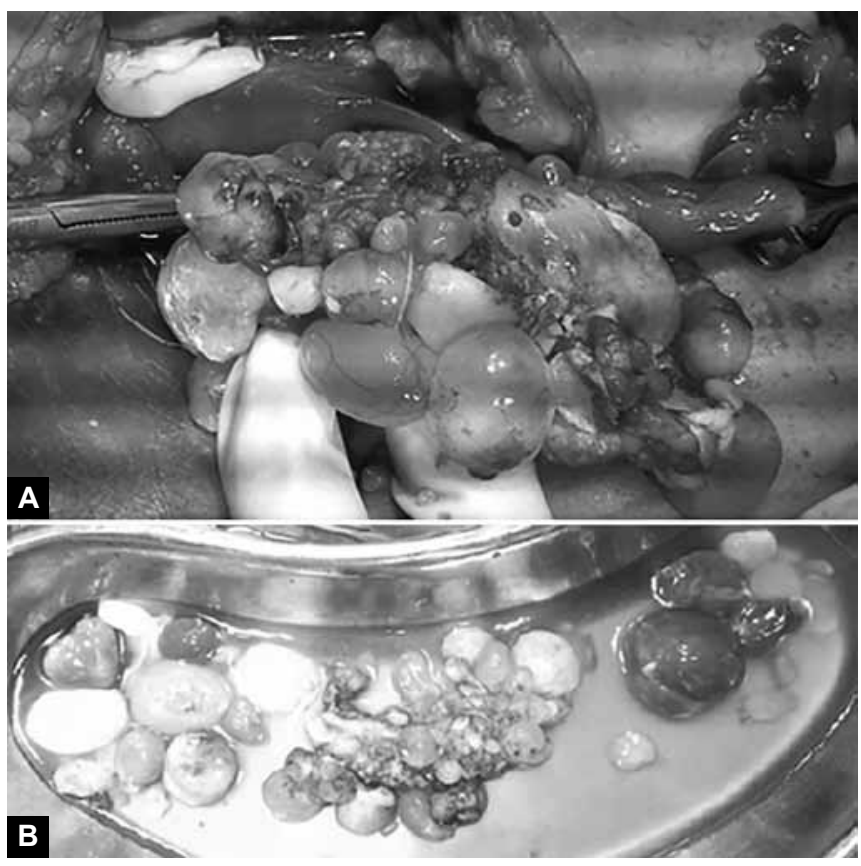

Figs 1A and B: (A) Laparoscopic finding showing multiple vesicles replacing ovarian tissue bilaterally as well as studded in omentum and peritoneal surfaces with normal uterus and bilateral patent fallopian tubes, and (B) multiple cysts separated from omental and peritoneal surfaces with specimen 


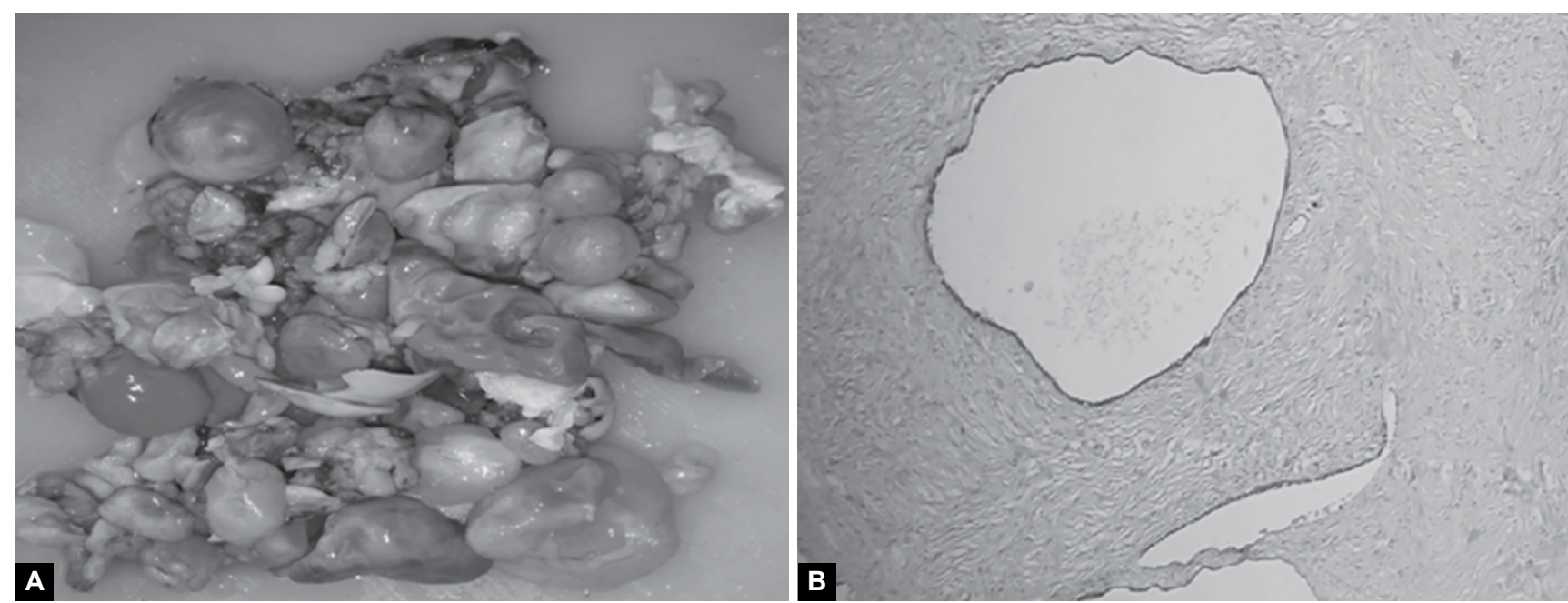

Figs 2A and B: (A) Grapes-like fluid-filled vesicular cysts of different colors and sizes after removal and (B) histopathological cut section showing cuboidal and columnar epithelium with mild atypia stroma fibrocollagenous

Peritoneal fluid cytology showed benign epithelial cells.

In post laparoscopy, her Ca-125 was $131.3(<35)$ and Ca-19.9 was $2(<27)$.

Her color Doppler was done which reported bilateral ovaries showing increased vascularity with normal spectral flow and velocity.

Her computed tomography (CT) scan was done which was suggestive of bilateral complex ovarian solid cystic mass in adnexa suggestive of cyst adenoma.

All these findings directed toward doubtful malignancy hence, laparotomy with frozen section was planned. On opening the abdomen, multiple free variable sized and variable colored cysts were found studded to omentum and peritoneal surface with their size ranging from $3 \mathrm{~mm}$ to $4 \mathrm{~cm}$.

Uterus was normal sized, with a smooth surface. Bilateral fallopian tubes were normal. Bilateral ovaries were replaced by multiple variable sized grape like fluid filled vesicles. Biopsy specimens were examined as frozen sections, leading to the diagnosis of benign cyst adenoma. Hence, decision of conservative surgery was taken.

Multiple moles like structures were removed leaving behind small amount of ovarian tissue bilaterally.

Histopathological (Figs 2A and B) examination was suggestive of serous cyst adenofibroma. Postoperative period was uneventful.

Spontaneous ovulation and intrauterine insemination (IUI) was planned for next cycle with 6 monthly follow-up to rule out reformation of cysts.

\section{DISCUSSION}

Due to rarity of tumor, very less literature was present in the textbooks.
Ideal postoperative management is not available anywhere. In few case reports extensive surgical excision was reported, which may be because of older age incidence of their cases. ${ }^{2}$

Cystadenofibroma of ovary is a type of surface epithelial tumor. These tumors exhibit a fibrous stroma in variable amounts in all subtypes. ${ }^{3}$ Ultrasonography (USG) cannot definitely characterize this tumor as its heterogenous appearance mimics a malignant ovarian neoplasm. A CT scan also is of limited value in evaluating this tumor. In a study by Cho et al, all 16 cases of ovarian cystadenofibromas, presenting as complex cystic masses with solid components, were preoperatively misdiagnosed as malignant ovarian neoplasms on CT scan or magnetic resonance imaging (MRI). ${ }^{1}$ Magnetic resonance imaging may be an essential modality for diagnosing this tumor, especially when the characteristic 'black sponge' appearance is observed on T2-weighted images. ${ }^{4}$

These masses are very often diagnosed as malignant on preoperative imaging, due to their solid component or irregular thick septae. Even at the time of surgery on gross examination, a cystadenofibroma may resemble a malignant tumor. That's why radical surgery is recommended in available text, but literature is still lacking in standard protocols for these tumors.

In this case, we preserved ovaries avoiding extensive surgical procedure as dilemma to treat infertility was there. We advised regular follow-up for spontaneous ovulation and IUI by husband, as effect of ovulation induction may be detrimental and prognosis could not be found anywhere in the available literature. 


\section{REFERENCES}

1. Cho SM, Byun JY, Rha SE et al. CT and MRI findings of cystadenofibromas of the ovary. Eur Radiol 2004;14(5):798-804.

2. Wasnik A, Elsayes K. Ovarian cystadenofibroma: A masquerade of malignancy. Ind J Radiol Imaging 2010 Nov; 20(4):297-299.
3. Lee DH. 'A Case of Mucinous Cystadenofibroma of the Ovary: case reports in obstetrics and gynecology 2014 Article ID 130530. doi:10.1155/2014/130530.

4. Byun JY. Magnetic resonance imaging findings of ovarian cystadenofibroma: clues for making the differential diagnosis from ovarian malignancy. Korean J Radiol 2006 Jul-Sep;7(3): 153-155. 